\title{
A high cell density perfusion process for MVA virus production: process integration with inline DNA digestion and cost analysis
}

\author{
Gwendal Gränicher ${ }^{1}$, Masoud Babakhani ${ }^{1}$, Sven Göbel ${ }^{1}$, Ingo Jordan ${ }^{2}$, Pavel \\ Marichal-Gallardo $^{1}$, Y Genzel ${ }^{1}$, and Udo Reichl ${ }^{1}$ \\ ${ }^{1}$ Max Planck Institute for Dynamics of Complex Technical Systems \\ ${ }^{2}$ ProBioGen AG
}

April 10, 2021

\begin{abstract}
By integrating continuous cell cultures with continuous purification methods, process yields and product quality attributes were improved over the last 10 years for recombinant protein production. However, for the production of viral vectors such as Modified Vaccinia virus Ankara (MVA), no such studies have been reported although there is an increasing need to meet the requirements for a rising number of clinical trials against infectious or neoplastic diseases. Here, we present for the first time a scalable suspension cell (AGE1.CR.pIX cells) culture-based perfusion process in bioreactors integrating continuous virus harvesting through an acoustic settler with semi-continuous chromatographic purification. This allowed to obtain purified MVA particles with a space-time yield $>600 \%$ higher for the integrated perfusion process (1.05 x 1011 TCID50/Lbioreactor/day) compared to the integrated batch process. Without further optimization, purification by membrane-based steric exclusion chromatography resulted in an overall product recovery of $50.5 \%$. To decrease the level of host cell DNA prior to chromatography, a novel inline continuous DNA digestion step was integrated into the process train. A detailed cost analysis comparing integrated production in batch versus production in perfusion mode showed that the cost per dose for MVA was reduced by nearly one third using this intensified small-scale process.
\end{abstract}

\section{Hosted file}

Paper integrated perfusion_21032021_Format.pdf available at https://authorea.com/users/ 407077/articles/517489-a-high-cell-density-perfusion-process-for-mva-virus-productionprocess-integration-with-inline-dna-digestion-and-cost-analysis 


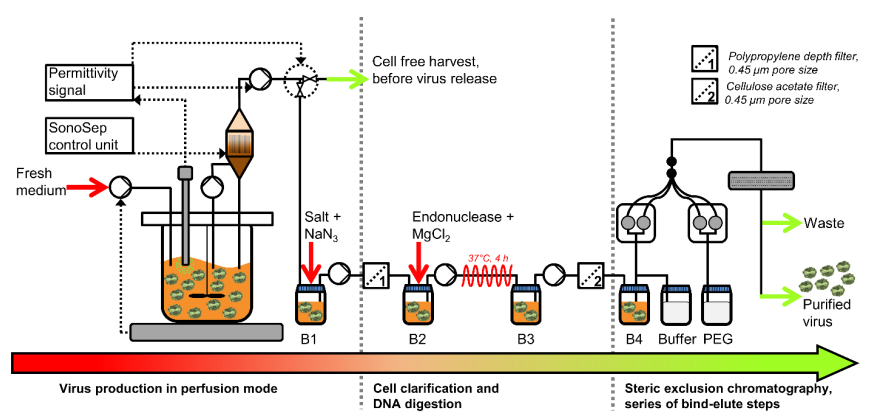

Figure 1: Scheme of an integrated process for cell culture-based virus production in perfusion mode. The integrated MVA production is separated in three main steps, separated by grey vertical dotted lines: 1 ) Virus production in perfusion mode using an acoustic filter, 2) cell clarification and DNA digestion, and 3) steric exclusion chromatography (SXC) as a series of bind-elute steps. MVA is produced using AGE1.CR.pIX cells grown in suspension in a stirred tank bioreactor. To achieve high cell concentrations, the cells are retained in the bioreactor while cell free medium is continuously harvested through the acoustic chamber controlled by the SonoSep control unit (acoustic filter as perfusion system). To allow a constant bioreactor working volume and weight, fresh medium is added into the bioreactor through a peristaltic pump controlled by a scale. During the cell growth phase, the harvest flow rate is controlled based on the estimation of the viable cell concentration using a capacitance sensor. After infection, a decrease in the permittivity signal indicates virus particle release, and initiates cell clarification and subsequent chromatography steps. The harvest containing MVA (which was first cell clarified through the acoustic settler) is collected into bottle B1. Salt and sodium azide (NaN3) are added to bottle B1 as well. The virus harvest is then continuously filtered through a polypropylene depth filter with $0.45 \mu \mathrm{m}$ pore size (Filter 1). For continuous endonuclease digestion (addition of endonuclease and magnesium chloride ( $\mathrm{MgCl} 2)$ in bottle B2), the harvest is incubated into a plug-flow reactor (indicated with the coiled red tube) at $37^{\circ} \mathrm{C}$ with a residence time of $4 \mathrm{~h}$. The endonuclease-digested product is continuously collected into bottle B3. After another filtration step using cellulose acetate depth filter with $0.45 \mu \mathrm{m}$ pore size (Filter 2), the harvest is collected into bottle B4 at $4^{\circ} \mathrm{C}$. An ÄKTA Pure 25 system is used to purify the virus harvest using membrane-based SXC operated in a semi-continuous bind-elute mode; the composition of buffer solutions (including buffer solution with PEG) used in purification are described in section 2.3.3. Finally, purified MVA is collected into $50 \mathrm{~mL}$ tubes (not illustrated). The color of the horizontal arrow going from red to green illustrates the stepwise purification of the MVA and the removal of contaminating host cell DNA. 

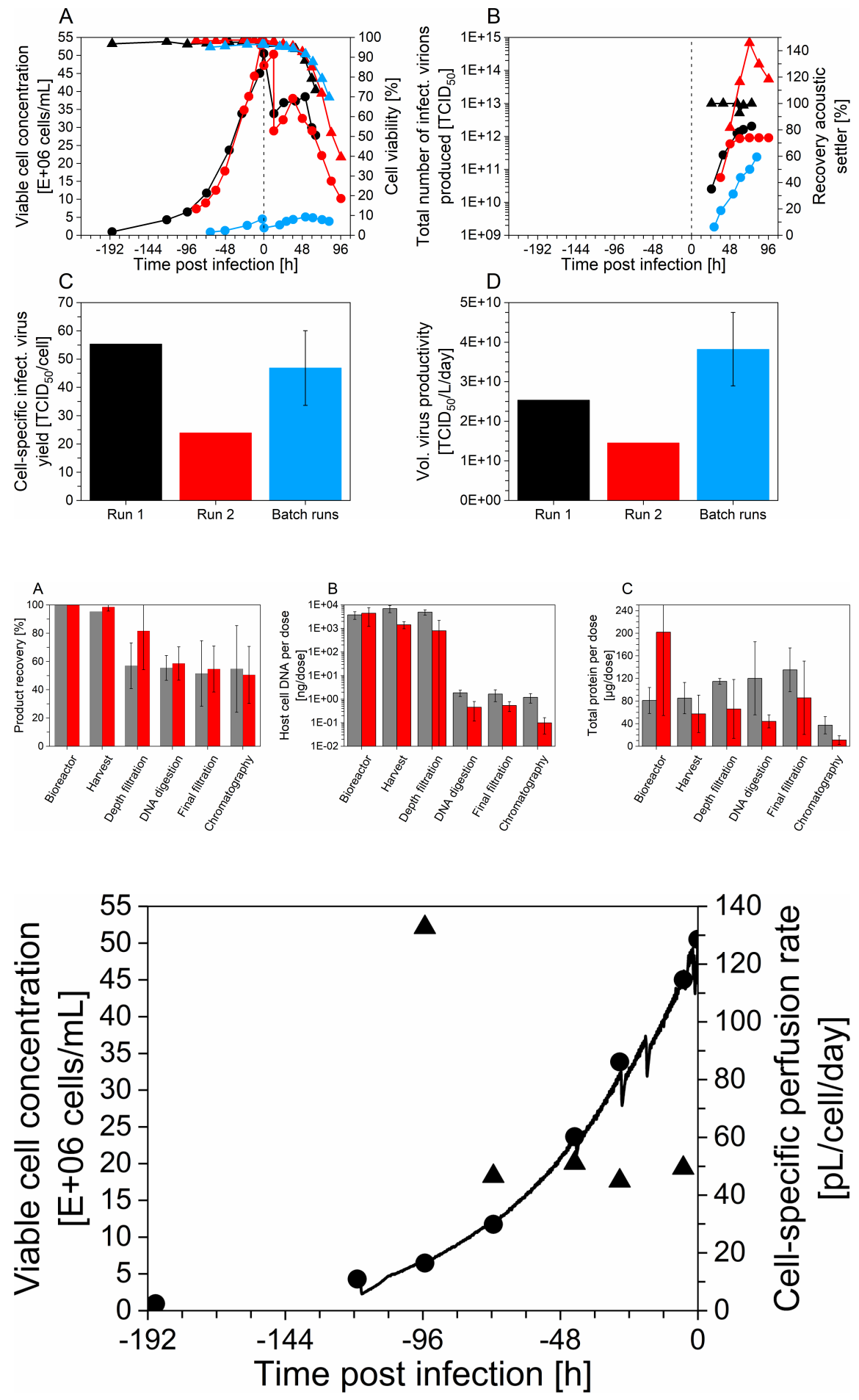

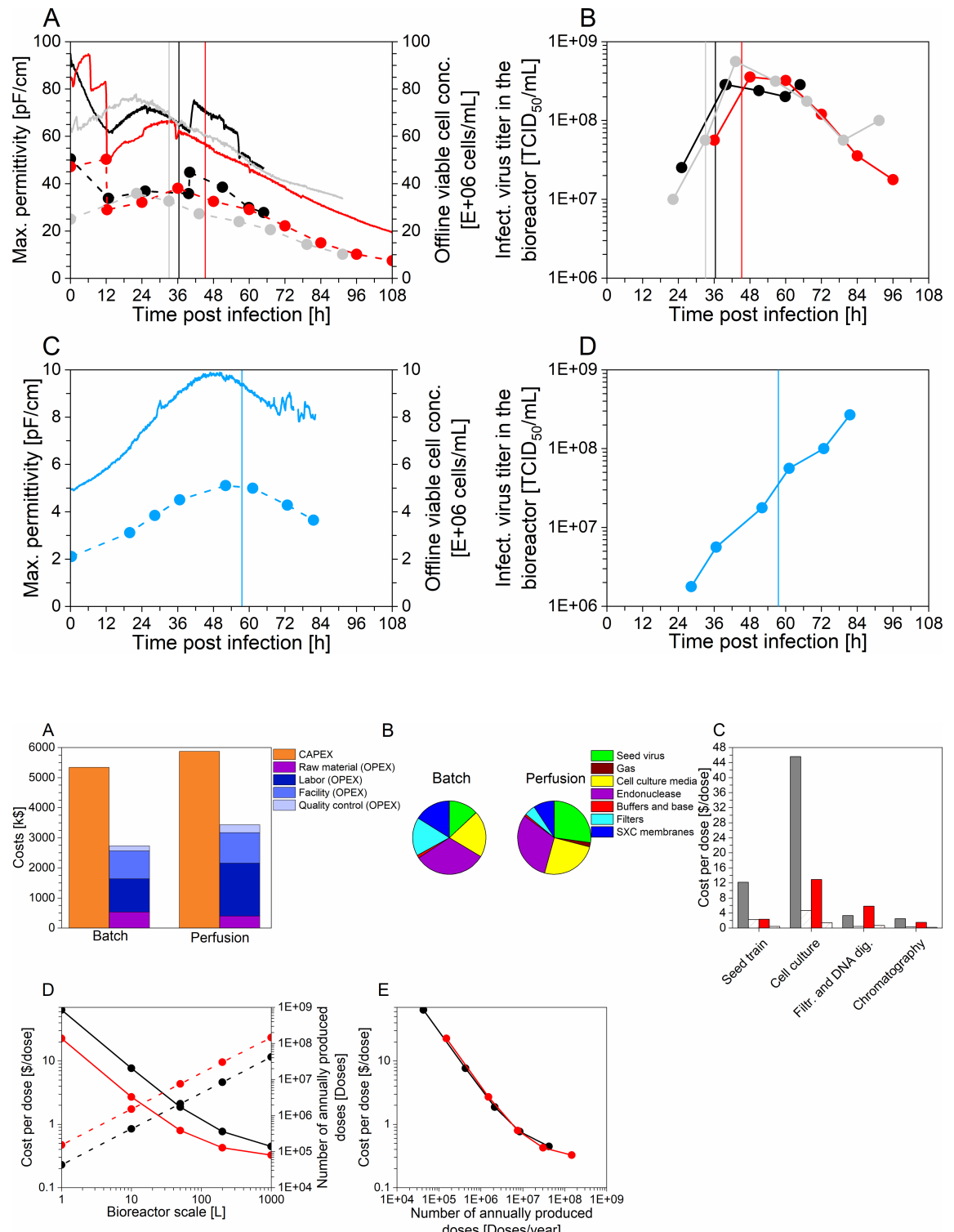


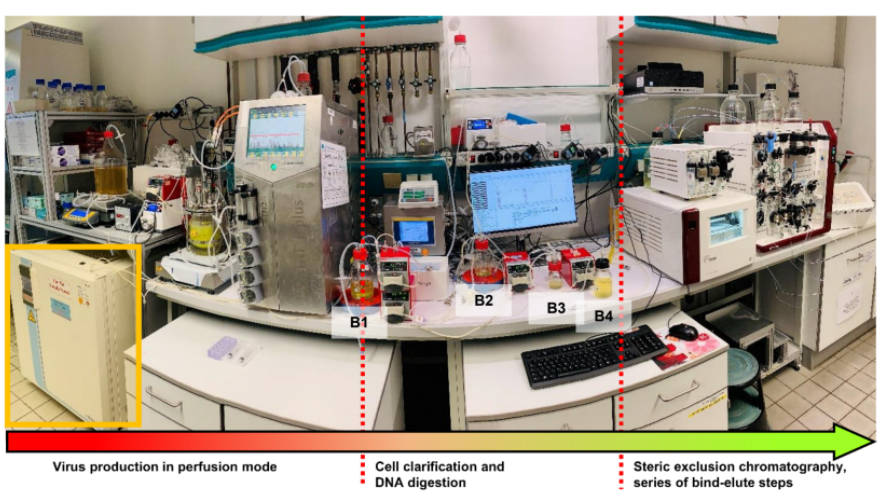

SUPPORTING INFORMATION FOR REVIEW AND PUBLICATION (APPENDICES)

Figure A.1: Picture of the process set-up, separated into three parts by red dashed vertical lines as shown in the scheme of Figure 1: 1) Virus production in the bioreactor (Biostat system coupled to an acoustic settler), 2) cell clarification and DNA digestion and 3) steric exclusion chromatography with an ÄKTA Pure 25 system operated in bind-elute mode. The plug flow reactor used for continuous DNA digestion at $37^{\circ} \mathrm{C}$ with a retention time of $4 \mathrm{~h}$ is located in the incubator, indicated by the orange square on the left side of the picture. The yellow color of the bioreactor and the bottles is due to the GFP protein expressed by the AGE1.CR.pIX cells after infection with the recombinant MVA.
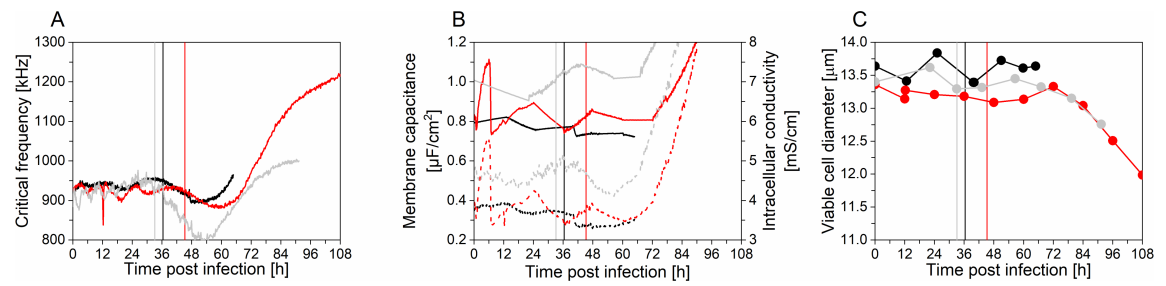


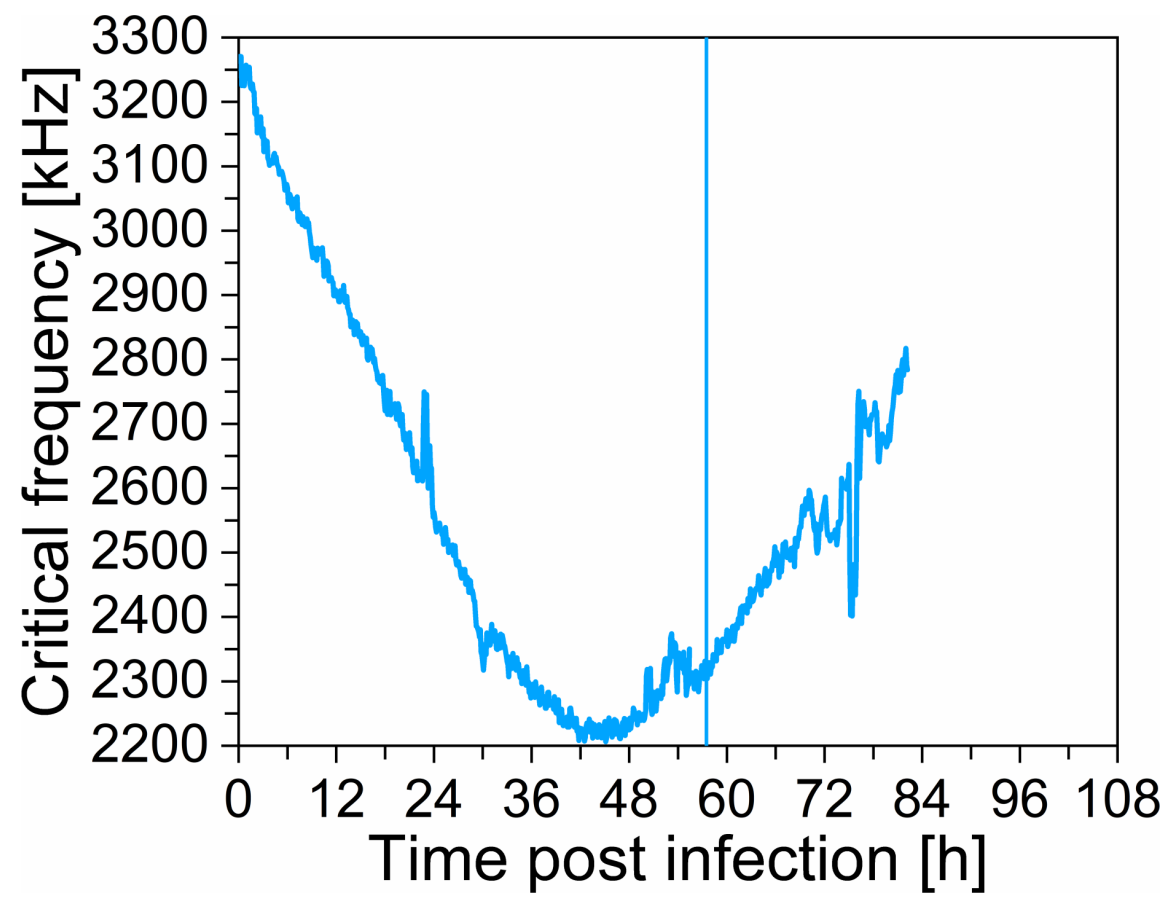

